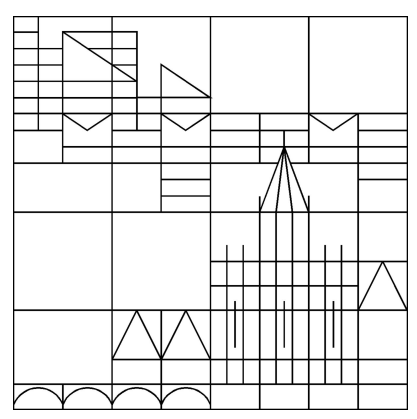

\title{
Global Attractors for Nonlinear Beam Equations
}

\author{
Reinhard Racke \\ Chanyu Shang
}

Konstanzer Schriften in Mathematik und Informatik

Nr. 249, Juni 2008

ISSN 1430-3558

Konstanzer Online-Publikations-System (KOPS)

URL: http://www.ub.uni-konstanz.de/kops/volltexte/2008/5687/

URN: http://nbn-resolving.de/urn:nbn:de:bsz:352-opus-56874

\footnotetext{
(C) Fachbereich Mathematik und Statistik

(C) Fachbereich Informatik und Informationswissenschaft

Universität Konstanz

Fach D 188, 78457 Konstanz, Germany

E-Mail: preprints@informatik.uni-konstanz.de

WWW: http://www.informatik.uni-konstanz.de/Schriften/
} 



\title{
Global Attractors for Nonlinear Beam Equations
}

\author{
Reinhard Racke and Chanyu Shang
}

\begin{abstract}
This paper is concerned with the dynamics for nonlinear one-dimensional beam equations. We consider a nonlinear beam equation with viscosity or with a lower order damping term instead of the viscosity, and we establish the existence of global attractors for both systems.
\end{abstract}

Keyword: beam equations; global attractor; infinite-dimensional dynamics

MSC 2000: 35G25; 35B41

\section{Introduction}

In this paper we investigate the existence of global attractors for the nonlinear onedimensional beam equations arising from the study of mechanical movements of shape memory alloys of constant mass density $\rho$ (assumed to be normalized to unity, i.e., $\rho=1$ ). We consider the equations either with viscosity, or without viscosity but with a lower order damping term, respectively. For both cases, our general aim, roughly stated, is to show that the equations possess global attractors in the corresponding complete metric spaces.

Let $\Omega=(0,1)$, and, for any $t>0, \Omega_{t}=\Omega \times(0, t)$. For the system with viscosity, the nonlinear partial differential equation we are studying is

$$
u_{t t}-\nu u_{x x t}-f\left(u_{x}\right)_{x}+R u_{x x x x}=g
$$

with $\mathrm{u}, \mathrm{f}, \mathrm{g}$ being the displacement, stess, density of distributed loads, respectively, and subject to the boundary conditions

$$
\left.u\right|_{x=0,1}=\left.u_{x x}\right|_{x=0,1}=0
$$

and the initial conditions

$$
\left.u\right|_{t=0}=u_{0},\left.\quad u_{t}\right|_{t=0}=u_{1}
$$


And for the system without viscosity, the equation we are studying is

$$
u_{t t}+\mu u_{t}-f\left(u_{x}\right)_{x}+R u_{x x x x}=g
$$

subject to the same boundary conditions (1.2) and initial conditions (1.3).

To study the thermomechanics of shape memory alloys in one space dimension, Falk [3], [4] has proposed a Ginzburg-Landau theory, using the strain $\varepsilon=u_{x}$ as order parameter and assuming that the Helmholtz free energy density F is a potential of Ginzburg-Landau form, i.e.,

$$
F=F\left(u_{x}, u_{x x}, \theta\right)
$$

where $\theta$ is the absolute temperature. Here the beam equations studied in our paper can be taken as the special case of [3], [4] for which with positive constant temperature. The simplest form for the free energy density $\mathrm{F}$, that accounts quite well for the experimentally behavior and takes couple stresses into account, is

$$
F\left(u_{x}, u_{x x}\right)=F_{1}\left(u_{x}\right)+\frac{R}{2} u_{x x}^{2}
$$

where

$$
F_{1}\left(u_{x}\right)=\frac{\alpha_{1}}{6} u_{x}^{6}-\frac{\alpha_{2}}{4} u_{x}^{4}-\frac{\alpha_{3}}{2} u_{x}^{2}
$$

with positive constants $\alpha_{i}$ and R.

The stress $f=f\left(u_{x}\right)$ in (1.1) or (1.4) is given by

$$
f\left(u_{x}\right)=F_{1}^{\prime}\left(u_{x}\right)=\alpha_{1} u_{x}^{5}-\alpha_{2} u_{x}^{3}-\alpha_{3} u_{x}
$$

and $\nu, \mu$ are positive constants.

The physical meaning of the boundary conditions is that both ends of the rod are hinged, respectively. For simplicity, we assume that $g \equiv 0$, i.e., no external force in the systems.

Before stating and proving our results, let us first recall some related results in the literature.

Ball [1] proved the existence of weak solutions to the nonlinear beam equation

$$
\frac{\partial^{2} u}{\partial t^{2}}+\alpha \frac{\partial^{4} u}{\partial x^{4}}-\left[\beta+\kappa \int_{0}^{l} u_{\xi}(\xi, t)^{2} d \xi\right] \frac{\partial^{2} u}{\partial x^{2}}=0
$$

subject to clamped or hinged boundary conditions. Later, Ball [2] proved the stability of an extensile beam equation as time tends to infinity. Eden and Milani [5] proved the 
existence of a compact attractor, and also an exponential attractor to the equations of the type

$$
\varepsilon u_{t t}+u_{t}+\alpha \Delta^{2} u=\left(\kappa \int_{\Omega}|\nabla u|^{2}-\beta\right) \Delta u+f .
$$

They also proved in the special case where damping is large, i.e., $\varepsilon$ is small, the exponential attractor contains the global attractor.

For the non-isothermal case, i.e., the coupled partial differential equations, which consist of a nonlinear beam equation with respect to the displacement $\mathrm{u}$ and a second order parabolic equation with respect to the temperature. Shang [12] proved the existence of a global attractor to the one-dimensional thermoviscoelastic system arising from the study of phase transitions in shape memory alloys with hinged boundary conditions in closed subspaces. Motivated by [12], equation (1.1) in our paper can be taken as the special case of [12] with constant temperature, but here we can proved the existence of a global attractor in the whole sobolev space H. For the same model as in [12], but with stress free boundary conditions at least at one end of the rod, Sprekels and Zheng [9] got the existence of a global attractor for the Ginzburg-Landau form for shape memory alloys. We can see that Shang [12], Sprekels and Zheng [9] studied the systems whose free energy density $\mathrm{F}$ was a potential of Ginzburg-Landau form, i.e., $R>0$. For the case $R=0, \nu>0$, Racke and Zheng [8] obtained the global existence and asymptotic behavior of the solution to the nonlinear thermoviscoelastic system with stress-free conditions at least at one end of the rod. For the system with clamped boundary conditions, Chen and Hoffmann [7] proved the global existence and uniqueness of the smooth solution. Shen, Zheng and Zhu [10] obtained the global existence and asymptotic behavior of the weak solution, and they established a new approach to derive a priori estimates on the $L^{\infty}$ norm of the strain $\mathrm{u}$ independent of the length of time. Recently, Qin, Liu and Song [11] obtained the existence of a global attractor for the same system as in [10].

In this paper, we consider problems (1.1)-(1.3) and (1.4)-(1.3). By deriving delicate uniform a priori estimates independent of $\mathrm{T}$ and the initial data for both cases, we obtain the results on the existence of global attractors.

First, we study the problem (1.1)-(1.3). Let

$$
H:=\left\{\left(u, u_{t}\right) \in H^{4} \times H^{2}:\left.u\right|_{x=0,1}=\left.u_{x x}\right|_{x=0,1}=0\right\}
$$

Our main result in this case reads as follows.

Theorem 1.1. Suppose $u_{0} \in H^{4}, u_{1} \in H^{2}$ are given functions that satisfy the compatibility conditions $\left.u_{0}\right|_{x=0,1}=\left.u_{0 x x}\right|_{x=0,1}=0$. Then for the problem (1.1)-(1.3) the following results hold. 
(i) The problem admits a unique global solution $\left(u, u_{t}\right)$ satisfying

$$
\begin{gathered}
u \in C\left([0,+\infty) ; H^{4}\right) \cap C^{1}\left([0,+\infty) ; H^{2}\right) \cap L^{2}\left([0,+\infty) ; H^{5}\right) ; \\
u_{t} \in C\left([0,+\infty) ; H^{2}\right) \cap L^{2}\left([0,+\infty) ; H^{3}\right) .
\end{gathered}
$$

(ii) The orbits starting from $H$ will reenter itself after finite time, and stay there forever. Moreover, it possesses in $H$ a global attractor $A$ which is compact.

Second, for the problem (1.4)-(1.3), our result is the following.

Theorem 1.2. Suppose $u_{0} \in H^{4}, u_{1} \in H^{2}$ are given functions that satisfy the compatibility conditions $\left.u_{0}\right|_{x=0,1}=\left.u_{0 x x}\right|_{x=0,1}=0$. Then for the problem (1.4)-(1.3) the following results hold.

(i)The problem admits a unique global solution $\left(u, u_{t}\right)$ satisfying

$$
\begin{gathered}
u \in C\left([0,+\infty) ; H^{4}\right) \cap C^{1}\left([0,+\infty) ; H^{2}\right) \cap L^{2}\left([0,+\infty) ; H^{5}\right) ; \\
u_{t} \in C\left([0,+\infty) ; H^{2}\right) \cap L^{2}\left([0,+\infty) ; H^{3}\right) .
\end{gathered}
$$

(ii) For $\beta>0$, we define the space

$$
H_{\beta}:=\left\{\left(u, u_{t}\right) \in H, \int_{0}^{1}\left(\frac{1}{2} u_{t}^{2}+\frac{R}{2} u_{x x}^{2}+F_{2}\left(u_{x}\right)\right) d x \leq \beta\right\}
$$

Then the orbits starting from $\mathrm{H}_{\beta}$ will reenter itself after finite time, and stay there forever. Moreover, it possesses in $H_{\beta}$ a global attractor $A_{\beta}$ which is compact.

In what follows, we explain some mathematical difficulties that appeared in this paper.

First, in the course of deriving the existence of an absorbing set in $\mathrm{H}$ or $H_{\beta}$, the estimates obtained in the proof of global existence are not sufficient, and we should derive uniform estimates of $\|u\|_{H^{4}},\left\|u_{t}\right\|_{H^{2}}$ independent of the initial data and t. It turns out more delicate estimates are needed due to the higher degree of nonlinearity inherent in the system and to the higher order derivative arising for $R>0$.

Second, we recall the results obtained in Eden and Milani [5], which followed a procedure similar to that of Hale [14], but replacing the role of the Lyapunov functions with different types of energy norms. Using the method of $\alpha$-contractions, [5] proved the existence of a compact, finite fractal dimensional invariant set toward which all solutions converged exponentially in time. However, the existence of global attractor, i.e., the boundedness of the attractor in the corresponding norm, could only be obtained when the damping is large, i.e., $\varepsilon$ is small in (1.9). Different from [5], in order to establish 
the existence of a global attractor, we shall apply the Theorem 6.4.1 in the book by Zheng [16]. The crucial step is to show the existence of an absorbing set and the uniform compactness of the orbits starting from any bounded set. In a similar manner to [16], we can obtain the existence of bounded, invariant absorbing set $B_{0}$ or $B_{\beta}$ for both cases. But in the proof of uniformly compactness, we can see that the problem (1.4)-(1.3), i.e., the system without viscosity, it seems to be totally different in comparison with the problem (1.1)-(1.3). The uniform compactness of the solution to the problem (1.4)-(1.3) can not be derived directly like the problem (1.1)-(1.3), due to the term $\mu u_{t}$ in (1.4) is not as good as $-\nu u_{x x t}$ in (1.1). In order to overcome this difficulty, we should rather consider the dynamics in closed subspaces defined by the parameter $\beta$, i.e., $H_{\beta}$ in our paper. We shall show that the constraint in the definition of $H_{\beta}$ is invariant under $\mathrm{S}(\mathrm{t})$. We shall prove that the orbit starting form $H_{\beta}$ will reenter itself after a finite time and stay there forever.

This paper is organized as follows. In section 2 we prove the existence of a global attractor for the problem (1.1)-(1.3) in the sobolev space H. In section 3 we prove the existence of a global attractor for the problem (1.4)-(1.3) in the closed subspace $H_{\beta}$.

The notation in this paper will be as follow : $L^{p}, W^{m, p}, 1 \leq p \leq \infty, m \in N$, $H^{1} \equiv W^{1,2}$, and $H_{0}^{1} \equiv W_{0}^{1,2}$, respectively, denote the usual Lebesgue and Sobolev space on $(0,1)$. We use the abbreviation $\|\cdot\|:=\|\cdot\|_{L^{2}}$, and $C^{k}(I, B), k \in N_{0}$, denote the space of k-times continuously differentiable functions from $I \in R$ into a Banach space B. The space $L^{p}(I, B), 1 \leq p \leq \infty$, are defined analogously. Finally, $\partial_{t}$ or subscript $\mathrm{t}$ and likewise, $\partial_{x}$ or a subscript $\mathrm{x}$, denote the partial derivations with respect to $\mathrm{t}$ and $\mathrm{x}$, respectively.

\section{The Existence of A Global Attractor for the System with Viscosity}

We consider the initial boundary value problem (1.1)-(1.3). In this section, we shall prove the existence of a global attractor for this system in the whole sobolev space $\mathrm{H}$.

We first establish a local existence and uniqueness result for this problem.

Lemma 2.1. Under the same assumption as in Theorem 1.1, there exists $t^{*}>0$ depending only on $\left\|u_{0}\right\|_{H^{4}(\Omega)}$, $\left\|u_{1}\right\|_{H^{2}(\Omega)}$, such that problem (1.1)-(1.3) admits a unique solution $\left(u, u_{t}\right)$ in $\bar{\Omega} \times\left[0, t^{*}\right]$ such that

$$
u \in C\left(\left[0, t^{*}\right] ; H^{4}\right) \cap C^{1}\left(\left[0, t^{*}\right] ; H^{2}\right) \cap L^{2}\left(\left[0, t^{*}\right] ; H^{5}\right),
$$




$$
u_{t} \in C\left(\left[0, t^{*}\right] ; H^{2}\right) \cap L^{2}\left(\left[0, t^{*}\right] ; H^{3}\right) .
$$

Proof. We use the contraction mapping theorem to prove the local existence and uniqueness. Since the proof is essentially the same as in Shang [12], we can omit the details here.

In the following we prove Theorem 1.1.

Proof of (i) in Theorem 1.1 In order to prove the global existence, we have to establish a priori estimates for $\|u\|_{H^{4}},\left\|u_{t}\right\|_{H^{2}}$. In fact, we can derive uniform a priori estimates independent of $t$, which is crucial for the proof of uniform compactness of the orbits. In this proof, the letter $\mathrm{C}$ denotes a universal positive constant that may depend on the norm of the initial data, but not on $t$.

Lemma 2.2. For any $t>0$, the following estimates hold.

$$
\begin{gathered}
\left\|u_{t}\right\| \leq C, \quad\left\|u_{x x}\right\| \leq C, \quad\left\|u_{x}\right\|_{L^{\infty}} \leq C, \\
\int_{0}^{t} \int_{0}^{1} u_{x t}^{2} d x d \tau \leq C, \quad \int_{0}^{t}\left\|u_{t}\right\|^{2} d \tau \leq C, \quad \int_{0}^{t}\left\|u_{t}\right\|_{L^{\infty}}^{2} d \tau \leq C .
\end{gathered}
$$

Proof. Multiplying (1.1) with $u_{t}$ and integrating with respect to $\mathrm{x}$ and t yields

$$
\frac{1}{2} \int_{0}^{1} u_{t}^{2} d x+\frac{R}{2} \int_{0}^{1} u_{x x}^{2} d x+\int_{0}^{1} F_{1}\left(u_{x}\right) d x+\nu \int_{0}^{t} \int_{0}^{1} u_{x t}^{2} d x d \tau \leq C .
$$

Here $F_{1}^{\prime}(x)=f(x)$, and applying Young's inequality, we have

$$
F_{1}\left(u_{x}\right) \geq C u_{x}^{6}-C
$$

Combining (2.3) with (2.4), we obtain the estimates (2.1). (2.2) can be derived form (2.1) and the boundary conditions (1.2) immediately. The proof is complete.

Lemma 2.3. For any $t>0$, the following estimates hold.

$$
\left\|u_{t t}\right\| \leq C, \quad\left\|u_{x x t}\right\| \leq C, \quad \int_{0}^{t}\left\|u_{x t t}\right\|^{2} d \tau \leq C, \quad \int_{0}^{t}\left\|u_{x x x t}\right\|^{2} d \tau \leq C .
$$

Proof. We differentiate (1.1) with respect to $t$, multiply the resultant by $u_{t t}$, and integrate with respect to $x$ over $\Omega$ to obtain

$$
\frac{1}{2} \frac{d}{d t} \int_{0}^{1} u_{t t}^{2} d x+\nu \int_{0}^{1} u_{x t t}^{2} d x+\int_{0}^{1} f\left(u_{x}\right)_{t} \cdot u_{x t t} d x+\frac{R}{2} \frac{d}{d t} \int_{0}^{1} u_{x x t}^{2} d x=0 .
$$

Since

$$
\int_{0}^{1} f\left(u_{x}\right)_{t} \cdot u_{x t t} d x \leq \frac{\nu}{2} \int_{0}^{1} u_{x t t}^{2} d x+C \int_{0}^{1}\left|f^{\prime}\left(u_{x}\right) u_{x t}\right|^{2} d x
$$




$$
\leq \frac{\nu}{2}\left\|u_{x t t}\right\|^{2}+C\left\|u_{x t}\right\|^{2} .
$$

Using (2.2) and integrating (2.6) with respect to t yields

$$
\left\|u_{t t}\right\| \leq C, \quad\left\|u_{x x t}\right\| \leq C, \quad \int_{0}^{t}\left\|u_{x t t}\right\|^{2} d \tau \leq C .
$$

Then we differentiate (1.1) with respect to $t$, multiply the resultant by $-u_{x x t}$, and integrate with respect to $x$ over $\Omega$ to obtain

$\frac{\nu}{2} \frac{d}{d t} \int_{0}^{1} u_{x x t}^{2} d x-\frac{d}{d t} \int_{0}^{1} u_{t t} \cdot u_{x x t} d x-\int_{0}^{1} u_{x t t}^{2} d x+R \int_{0}^{1} u_{x x x t}^{2} d x-\int_{0}^{1} f\left(u_{x}\right)_{t} \cdot u_{x x x t} d x=0$

Using the estimates we obtain in (2.8), we have

$$
\begin{aligned}
\int_{0}^{1} f\left(u_{x}\right)_{t} \cdot u_{x x x t} d x & \leq \frac{R}{2} \int_{0}^{1} u_{x x x t}^{2} d x+C \int_{0}^{1}\left|f\left(u_{x}\right)_{t}\right|^{2} d t \\
& \leq \frac{R}{2}\left\|u_{x x x t}\right\|^{2} d x+C .
\end{aligned}
$$

Combing (2.9) with (2.10), we finally have

$$
\int_{0}^{t}\left\|u_{x x x t}\right\|^{2} d \tau \leq C
$$

The proof is complete.

Having established uniform a priori estimates, the global existence and uniqueness follows from the continuation argument. In what follows, we will prove the compactness of the orbit for $t>0$ in $H^{4} \times H^{2}$. For the time being, we assume that the initial data are so smooth that the solution will have enough smoothness to carry out the following argument. If the initial data just belong to $H^{4} \times H^{2}$, we can approximate them by smooth functions and then pass to the limit.

Lemma 2.4. For any $\mu>0$, the triple $\left(u, u_{t}\right)$ is bounded in $C\left([\mu,+\infty) ; H^{5} \times H^{3}\right)$.

Proof. First, we differentiate (1.1) with respect to $t$, multiply the resultant by $-u_{x x t t}$, and integrate with respect to $x$ over $\Omega$ to obtain

$$
\frac{d}{d t} \int_{0}^{1}\left(\frac{R}{2} u_{x x x t}^{2}+\frac{1}{2} u_{x t t}^{2}\right) d x+\frac{\nu}{2} \int_{0}^{1} u_{x x t t}^{2} d x \leq C \int_{0}^{1}\left|f\left(u_{x}\right)_{x t}\right|^{2} d x .
$$

Multiplying (2.12) by t, we obtain

$\frac{d}{d t}\left(t R\left\|u_{x x x t}\right\|^{2}+t\left\|u_{x t t}\right\|^{2}\right)+\nu t\left\|u_{x x t t}\right\|^{2} \leq\left(R\left\|u_{x x x t}\right\|^{2}+\left\|u_{x t t}\right\|^{2}\right)+C t\left\|f\left(u_{x}\right)_{x t}\right\|^{2}$. 
Since

$$
\int_{0}^{t}\left\|f\left(u_{x}\right)_{x t}\right\|^{2} d \tau=\int_{0}^{t}\left(\left\|f^{\prime}\left(u_{x}\right) u_{x x t}\right\|^{2}+\left\|f^{\prime \prime}\left(u_{x}\right) u_{x x} u_{x t}\right\|^{2}\right) d \tau .
$$

Using Nirenberg's inequality, we have

$$
\left\|u_{x x t}\right\| \leq C\left\|u_{x x x t}\right\|^{\frac{1}{2}} \cdot\left\|u_{x t}\right\|^{\frac{1}{2}}
$$

and Young's inequality,

$$
\left\|u_{x x t}\right\|^{2} \leq C\left\|u_{x x x t}\right\| \cdot\left\|u_{x t}\right\| \leq \frac{C}{2}\left\|u_{x x x t}\right\|^{2}+\frac{C}{2}\left\|u_{x t}\right\|^{2} .
$$

Combining with the estimates in Lemma 2.3 yields

$$
\int_{0}^{t}\left\|u_{x x t}\right\|^{2} d \tau \leq C
$$

Similarly, since

$$
\int_{0}^{t}\left\|u_{x x} u_{x t}\right\|^{2} d \tau \leq \int_{0}^{t}\left\|u_{x t}\right\|_{L^{\infty}}^{2} \cdot\left\|u_{x x}\right\|^{2} d \tau \leq C \int_{0}^{t}\left\|u_{x t}\right\|_{L^{\infty}}^{2} d \tau
$$

and

$$
\left\|u_{x t}\right\|_{L^{\infty}}^{2} \leq C\left\|u_{x x x t}\right\|^{\frac{1}{2}} \cdot\left\|u_{x t}\right\|^{\frac{3}{2}} \leq \frac{C}{2}\left\|u_{x x x t}\right\|^{2}+\frac{C}{2}\left\|u_{x t}\right\|^{2} .
$$

Thus,

$$
\int_{0}^{t}\left\|u_{x t}\right\|_{L^{\infty}}^{2} d \tau \leq C
$$

Finally, we obtain

$$
\int_{0}^{t}\left\|f\left(u_{x}\right)_{x t}\right\|^{2} d \tau \leq C
$$

Thus we can get from $(2.13)$

$$
R\left\|u_{x x x t}\right\|^{2}+\left\|u_{x t t}\right\|^{2} \leq \tilde{C} t^{-1}+C
$$

with $\tilde{C}=\tilde{C}\left(\left\|u_{0}\right\|_{H^{4}},\left\|u_{1}\right\|_{H^{2}}\right)$. The proof is complete.

From this Lemma the compactness of the orbit in $H^{4} \times H^{2}$ follows. In what follows, we shall prove (ii) of Theorem 1.1, i.e., the existence of a global attractor in $\mathrm{H}$.

Proof of (ii) in Theorem 1.1 In order to prove the existence of a global attractor, we shall apply Theorem I.1.1 in the book by Temam [14], which Shen and Zheng [6] rephrased as follows. 
Theorem 2.1. Suppose that

(a) the mapping $S(t), t \geq 0$ defined by the solution to problem (1.1)-(1.3) is a nonlinear continuous semigroup from $H$ into itself and is uniformly compact for $t$ large;

(b) there exists a bounded set $B$ in $H$ such that $B$ is absorbing in $H$.

Then the $\omega$-limit set of $B$ is a global attractor which is compact and attracts the bounded sets of $H$.

Concerning (a), we have proved in Theorem 1.1 (i) the global existence of the solution. It is clear from the proof that the family of operators $S(t), t \geq 0$ defined by the solution are continuous operators from $\mathrm{H}$ to $\mathrm{H}$ and they enjoy the usual semigroup properties. The uniform compactness of the orbit has been proved in Lemma 2.4. Hence, what remains is to verify the condition (b). In the following, the letters $\mathrm{C}, C_{i}$ denote positive constants independent of the initial data and the time t.

Let $B_{0}=\left\{\left(u, u_{t}\right) \in H, \quad\|u\|_{H^{4}} \leq \bar{C}_{1}, \quad\left\|u_{t}\right\|_{H^{2}} \leq \bar{C}_{2}\right\}$ where $\bar{C}_{1}, \bar{C}_{2}$ are also positive constants independent of the initial data and $t$, which will be specified later. Then we have

Lemma 2.5. $B_{0}$ is an absorbing set in $H$, i.e., for any bounded set $B$ in $H$, there exists some time $t_{2}=t_{2}(B)>0$, such that when $t \geq t_{2}(B), S(t) B \subset B_{0}$.

Proof. Multiplying (1.1) with $u$ and integrating with respect to x yields

$$
\frac{d}{d t} \int_{0}^{1} u u_{t} d x-\int_{0}^{1} u_{t}^{2} d x+\frac{\nu}{2} \frac{d}{d t} \int_{0}^{1} u_{x}^{2} d x+\int_{0}^{1} f\left(u_{x}\right) \cdot u_{x} d x+R \int_{0}^{1} u_{x x}^{2} d x=0 .
$$

Using Young's inequality, we obtain

$$
f\left(u_{x}\right) \cdot u_{x} \geq C u_{x}^{6}-C .
$$

Multiplying (1.1) with $u_{t}$ and integrating with respect to $\mathrm{x}$ yields

$$
\frac{1}{2} \frac{d}{d t} \int_{0}^{1} u_{t}^{2} d x+\frac{R}{2} \frac{d}{d t} \int_{0}^{1} u_{x x}^{2} d x+\frac{d}{d t} \int_{0}^{1} F_{1}\left(u_{x}\right) d x+\nu \int_{0}^{1} u_{x t}^{2} d x=0,
$$

here

$$
F_{1}\left(u_{x}\right) \geq C u_{x}^{6}-C .
$$

Using Poincare's inequality and the boundary condition (1.2), we have

$$
\left\|u_{t}\right\|_{L^{2}} \leq\left\|u_{t}\right\|_{L^{\infty}} \leq\left\|u_{x t}\right\|_{L^{2}}
$$

Now, we multiply (2.15) by $\frac{\nu}{2}$ and add the resultant to (2.16) to obtain

$$
\frac{d}{d t}\left(\frac{\nu}{2} \int_{0}^{1} u u_{t}+\frac{\nu^{2}}{4} \int_{0}^{1} u_{x}^{2} d x+\frac{1}{2} \int_{0}^{1} u_{t}^{2} d x+\frac{R}{2} \int_{0}^{1} u_{x x}^{2} d x+\int_{0}^{1} u_{x}^{6} d x\right)
$$




$$
+\frac{\nu}{2} \int_{0}^{1} u_{x}^{6} d x+\frac{\nu R}{2} \int_{0}^{1} u_{x x}^{2} d x+\frac{\nu}{2} \int_{0}^{1} u_{x t}^{2} d x \leq C .
$$

If we define

$$
E_{1}(t):=\frac{\nu}{2} \int_{0}^{1} u u_{t}+\frac{\nu^{2}}{4} \int_{0}^{1} u_{x}^{2} d x+\frac{1}{2} \int_{0}^{1} u_{t}^{2} d x+\frac{R}{2} \int_{0}^{1} u_{x x}^{2} d x+\int_{0}^{1} u_{x}^{6} d x
$$

and

$$
E_{2}(t):=\frac{\nu}{2} \int_{0}^{1} u_{x}^{6} d x+\frac{\nu R}{2} \int_{0}^{1} u_{x x}^{2} d x+\frac{\nu}{2} \int_{0}^{1} u_{x t}^{2} d x .
$$

Using Poincare's inequality and the boundary conditions again, we have

$$
E_{1}(t) \sim\|u\|_{H^{2}}^{2}+\left\|u_{t}\right\|_{L^{2}}^{2}
$$

and

$$
E_{1}(t) \leq C E_{2}(t)
$$

Thus, we have

$$
\frac{d E_{1}(t)}{d t}+C_{1} E_{1}(t) \leq C_{2}
$$

then it leads to

$$
E_{1}(t) \leq E_{1}(0) e^{-C_{1} t}+\frac{C_{2}}{C_{1}}
$$

We can see from (2.19) that for any initial data starting from any bounded set B of $\mathrm{H}$, there exists $t_{1}(B)$, such that when $t \geq t_{1}(B)$,

$$
E_{1}(t) \leq \frac{2 C_{2}}{C_{1}}
$$

In what follows, we consider the solution in $\left[t_{1}(B),+\infty\right)$. From $(2.20)$, we have

$$
\left\|u_{t}\right\|^{2} \leq \frac{2 C_{2}}{C_{1}}, \quad\left\|u_{x x}\right\|^{2} \leq \frac{2 C_{2}}{C_{1}}, \text { for any } t \geq t_{1}(B)
$$

and

$$
\left\|u_{x}\right\|_{L^{\infty}}^{n+2} \leq\left\|u_{x x}\right\|_{L^{2}}^{n+2} \leq\left(\frac{2 C_{2}}{C_{1}}\right)^{\frac{n+2}{2}}
$$

Differentiating (1.1) with respect to $t$, multiplying the resultant by $u_{t t}$, and integrating with respect to $x$ over $\Omega$ to obtain

$$
\frac{1}{2} \frac{d}{d t} \int_{0}^{1} u_{t t}^{2} d x+\nu \int_{0}^{1} u_{x t t}^{2} d x+\frac{R}{2} \frac{d}{d t} \int_{0}^{1} u_{x x t}^{2} d x=-\int_{0}^{1} f\left(u_{x}\right)_{t} \cdot u_{x t t} d x .
$$

Differentiating (1.1) with respect to $t$, multiplying the resultant by $-u_{x x t}$, and integrating with respect to $x$ over $\Omega$ to obtain

$$
\frac{\nu}{2} \frac{d}{d t} \int_{0}^{1} u_{x x t}^{2} d x-\frac{d}{d t} \int_{0}^{1} u_{t t} \cdot u_{x x t} d x-\int_{0}^{1} u_{x t t}^{2} d x+R \int_{0}^{1} u_{x x x t}^{2} d x=\int_{0}^{1} f\left(u_{x}\right)_{t} \cdot u_{x x x t} d x
$$


In the following, we estimate the right-hand side of (2.23), (2.24) respectively.

$$
\int_{0}^{1} f\left(u_{x}\right)_{t} \cdot u_{x t t} d x \leq \frac{\nu}{4} \int_{0}^{1} u_{x t t}^{2} d x+C \int_{0}^{1} f\left(u_{x}\right)_{t}^{2} d x
$$

Observe that

$$
\int_{0}^{1} f\left(u_{x}\right)_{t}^{2} d x=\int_{0}^{1}\left|f^{\prime}\left(u_{x}\right) \cdot u_{x t}\right|^{2} d x \leq C \int_{0}^{1} u_{x}^{8} u_{x t}^{2} d x+C \int_{0}^{1} u_{x t}^{2} d x .
$$

By virtue of the previous estimates,

$$
\int_{0}^{1} u_{x}^{8} u_{x t}^{2} d x \leq\left\|u_{x}\right\|_{L^{\infty}}^{8}\left\|u_{x t}\right\|_{L^{2}}^{2} \leq\left(\frac{2 C_{2}}{C_{1}}\right)^{4}\left\|u_{x t}\right\|_{L^{2}}^{2}
$$

and

$$
\left\|u_{x t}\right\|_{L^{2}}^{2} \leq C\left\|u_{x x x t}\right\|_{L^{2}}^{\frac{2}{3}} \cdot\left\|u_{t}\right\|_{L^{2}}^{\frac{4}{3}} \leq \delta\left\|u_{x x x t}\right\|_{L^{2}}^{2}+C_{\delta}\left\|u_{t}\right\|_{L^{2}}^{2}
$$

with $\delta$ being a positive constant. Thus,

$$
\int_{0}^{1} u_{x}^{8} u_{x t}^{2} d x \leq \delta\left\|u_{x x x t}\right\|_{L^{2}}^{2}+C_{\delta}\left\|u_{t}\right\|_{L^{2}}^{2}
$$

Similarly, we have

$$
\int_{0}^{1} f\left(u_{x}\right)_{t} \cdot u_{x x x t} d x \leq \delta\left\|u_{x x x t}\right\|_{L^{2}}^{2}+C_{\delta}\left\|u_{t}\right\|_{L^{2}}^{2}
$$

Multiplying (2.24) by $\eta$ and adding the resultant to (2.23) yields

$$
\begin{aligned}
& \frac{d}{d t}\left(\frac{1}{2} \int_{0}^{1} u_{t t}^{2} d x+\left(\frac{R}{2}+\frac{\nu \eta}{2}\right) \int_{0}^{1} u_{x x t}^{2} d x-\eta \int_{0}^{1} u_{t t} \cdot u_{x x t} d x\right) \\
& +(\nu-\eta) \int_{0}^{1} u_{x t t}^{2} d x+R \eta \int_{0}^{1} u_{x x x t}^{2} d x \leq \delta\left\|u_{x x x t}\right\|^{2}+C_{\delta}\left\|u_{t}\right\|^{2} .
\end{aligned}
$$

We can choose $\eta, \delta$ small enough to make sure the positivity of the coefficients on the left-hand of (2.30). Then we obtain

$$
\frac{d}{d t}\left(\int_{0}^{1} u_{t t}^{2} d x+\int_{0}^{1} u_{x x t}^{2} d x\right)+C_{3}\left(\int_{0}^{1} u_{x t t}^{2} d x+\int_{0}^{1} u_{x x x t}^{2} d x\right) \leq C_{4} .
$$

Combining with (2.18), we finally have

$$
\begin{aligned}
& \frac{d}{d t}\left(\int_{0}^{1} u u_{t} d x+\int_{0}^{1} u_{x}^{2} d x+\int_{0}^{1} u_{t}^{2} d x+\int_{0}^{1} u_{x x}^{2} d x+\int_{0}^{1} u_{x}^{6} d x+\int_{0}^{1} u_{t t}^{2} d x+\int_{0}^{1} u_{x x t}^{2} d x\right) \\
& +C_{5}\left(\int_{0}^{1} u_{x}^{6} d x+\int_{0}^{1} u_{x x}^{2} d x+\int_{0}^{1} u_{x t}^{2} d x+\int_{0}^{1} u_{x t t}^{2} d x+\int_{0}^{1} u_{x x x t}^{2} d x\right) \leq C_{6} .
\end{aligned}
$$

If we define

$$
E_{3}(t):=\int_{0}^{1} u u_{t} d x+\int_{0}^{1} u_{x}^{2} d x+\int_{0}^{1} u_{t}^{2} d x+\int_{0}^{1} u_{x x}^{2} d x+\int_{0}^{1} u_{x}^{6} d x+\int_{0}^{1} u_{t t}^{2} d x+\int_{0}^{1} u_{x x t}^{2} d x
$$


and

$$
E_{4}(t):=\int_{0}^{1} u_{x}^{6} d x+\int_{0}^{1} u_{x x}^{2} d x+\int_{0}^{1} u_{x t}^{2} d x+\int_{0}^{1} u_{x t t}^{2} d x+\int_{0}^{1} u_{x x x t}^{2} d x,
$$

then using Poincare's inequality and the boundary condition 1.2, we have

$$
\begin{gathered}
E_{3}(t) \sim\|u\|_{H^{4}}^{2}+\left\|u_{t}\right\|_{H^{2}}^{2}, \\
E_{3}(t) \leq C E_{4}(t) .
\end{gathered}
$$

Similarly as in the estimates of $E_{1}(t)$, we have

$$
\frac{d E_{3}(t)}{d t}+C_{7} E_{3}(t) \leq C_{8}, \text { for any } t \geq t_{1}(B)
$$

which immediately leads to

$$
E_{3}(t) \leq E_{3}(0) e^{-C_{7} t}+\frac{C_{8}}{C_{7}}, \text { for any } t \geq t_{1}(B) .
$$

For the initial data starting from the bounded set B mentioned above, there exists $t_{2}(B) \geq$ $t_{1}(B)$, such that when $t \geq t_{2}(B)$, we have

$$
E_{3}(t) \leq \frac{2 C_{8}}{C_{7}}
$$

From (2.34), we can see that if we choose $\bar{C}_{1}=\bar{C}_{2}=\frac{2 C_{8}}{C_{7}}$ in the definition of $B_{0}$, the existence of absorbing set $B_{0}$ follows. The proof is complete.

\section{The Existence of A Global Attractor for the System without Viscosity}

We consider the initial boundary value problem (1.4)-(1.3). In this section, we shall prove the existence of a global attractor for this system in the closed subspace $H_{\beta}$.

Here we define $H_{\beta}$ as

$$
H_{\beta}:=\left\{\left(u, u_{t}\right) \in H, \int_{0}^{1}\left(\frac{1}{2} u_{t}^{2}+\frac{R}{2} u_{x x}^{2}+F_{1}\left(u_{x}\right)\right) d x \leq \beta\right\} .
$$

We establish the local existence and uniqueness results in a similar way to section 2.

Lemma 3.1. Under the same assumption as in Theorem 1.2. There exists $t^{*}>0$ depending only on $\left\|u_{0}\right\|_{H^{4}(\Omega)}$, $\left\|u_{1}\right\|_{H^{2}(\Omega)}$, such that problem (1.4)-(1.3) admits a unique solution $\left(u, u_{t}\right)$ in $\bar{\Omega} \times\left[0, t^{*}\right]$ such that

$$
\begin{gathered}
u \in C\left(\left[0, t^{*}\right] ; H^{4}\right) \cap C^{1}\left(\left[0, t^{*}\right] ; H^{2}\right) \cap L^{2}\left(\left[0, t^{*}\right] ; H^{5}\right), \\
u_{t} \in C\left(\left[0, t^{*}\right] ; H^{2}\right) \cap L^{2}\left(\left[0, t^{*}\right] ; H^{3}\right) .
\end{gathered}
$$


Proof of (i) in Theorem 1.2. We can only obtain a priori estimates depending on T. In what follows, the letter $C_{T}$ denotes a positive constant which may depend on the initial data and the time $\mathrm{T}$.

Lemma 3.2. For any $t \in[0, T]$, the following estimates hold.

$$
\left\|u_{t}\right\| \leq C_{T}, \quad\left\|u_{x x}\right\| \leq C_{T}, \quad\left\|u_{x}\right\|_{L^{\infty}} \leq C_{T}, \quad \int_{0}^{t}\left\|u_{t}\right\|^{2} d \tau \leq C_{T}
$$

Proof. Multiplying (1.4) by $u_{t}$ and integrating with respect to $\mathrm{x}$ yields

$$
\frac{d}{d t}\left(\frac{1}{2} \int_{0}^{1} u_{t}^{2} d x+\frac{R}{2} \int_{0}^{1} u_{x x}^{2} d x+\int_{0}^{1} F\left(u_{x}\right) d x\right)+\mu \int_{0}^{1} u_{t}^{2} d x=0 .
$$

From (3.2), the estimates of (3.1) follow immediately.

Lemma 3.3. For any $t \in[0, T]$, the following estimates hold.

$$
\left\|u_{t t}\right\| \leq C_{T}, \quad\left\|u_{x x t}\right\| \leq C_{T}
$$

Proof. We differentiate (1.4) with respect to $t$, multiply the resultant by $u_{t t}$, and integrate with respect to $x$ over $\Omega$ to obtain

$$
\frac{1}{2} \frac{d}{d t} \int_{0}^{1} u_{t t}^{2} d x+\mu \int_{0}^{1} u_{t t}^{2} d x+\int_{0}^{1} f\left(u_{x}\right)_{t} \cdot u_{x t t} d x+\frac{R}{2} \frac{d}{d t} \int_{0}^{1} u_{x x t}^{2} d x=0
$$

Since

$$
\int_{0}^{1} f\left(u_{x}\right)_{x t} \cdot u_{t t} d x \leq \frac{\mu}{2} \int_{0}^{1} u_{t t}^{2} d x+C_{\mu} \int_{0}^{1}\left|f\left(u_{x}\right)_{x t}\right|^{2} d x
$$

and

$$
\begin{aligned}
\int_{0}^{1}\left|f\left(u_{x}\right)_{x t}\right|^{2} d x & =\int_{0}^{1}\left|f^{\prime \prime}\left(u_{x}\right) u_{x x} u_{x t}\right|^{2} d x+\int_{0}^{1}\left|f^{\prime}\left(u_{x}\right) u_{x x t}\right|^{2} d x \\
& \leq C\left\|u_{x t}\right\|^{2}+C\left\|u_{x x t}\right\|^{2} \\
& \leq C\left\|u_{x x t}\right\|^{2}+C
\end{aligned}
$$

here

$$
\left\|u_{x t}\right\|^{2} \leq C\left\|u_{x x t}\right\|^{2}+C\left\|u_{t}\right\|^{2}
$$

Applying Gronwall's inequality, we can obtain

$$
\left\|u_{t t}\right\| \leq C_{T}, \quad\left\|u_{x x t}\right\| \leq C_{T}
$$

The proof is complete. 
Combing Lemma 3.2 with equation (1.4), we can obtain the boundedness of $\|u\|_{H^{4}}$, $\left\|u_{t}\right\|_{H^{2}}$, then the global existence and uniqueness follows.

Proof of (ii) in Theorem 1.2. First, we prove the existence of an absorbing set in $H_{\beta}$. In the following, letter $\mathrm{C}$ and $C_{i}$ denote positive constants depending only on $\beta$.

Let $B_{\beta}=\left\{\left(u, u_{t}\right) \in H_{\beta}, \quad\|u\|_{H^{4}} \leq \bar{C}_{1}, \quad\left\|u_{t}\right\|_{H^{2}} \leq \bar{C}_{2}\right\}$ where $\bar{C}_{1}, \bar{C}_{2}$ are positive constants that may depend on $\beta$, but not on the initial data and $\mathrm{t}$, and they will be specified later. Then we have

Lemma 3.4. $B_{\beta}$ is an absorbing set in $H_{\beta}$, i.e., for any bounded set $B$ in $H_{\beta}$, there exists some time $t=t_{0}(B)>0$, such that when $t \geq t_{0}(B), S(t) B \subset B_{\beta}$.

Proof. From now on, we assume that the initial data $\left(u_{0}, u_{1}\right) \in B \subset H_{\beta}$.

First, we multiply (1.4) by $u_{t}$ and integrate with respect to $\mathrm{x}$ to obtain

$$
\frac{d}{d t}\left(\frac{1}{2} \int_{0}^{1} u_{t}^{2} d x+\frac{R}{2} \int_{0}^{1} u_{x x}^{2} d x+\int_{0}^{1} F\left(u_{x}\right) d x\right)+\mu \int_{0}^{1} u_{t}^{2} d x=0,
$$

then we have

$$
\begin{aligned}
& \frac{1}{2} \int_{0}^{1} u_{t}^{2} d x+\frac{R}{2} \int_{0}^{1} u_{x x}^{2} d x+\int_{0}^{1} F\left(u_{x}\right) d x \\
& \leq \frac{1}{2} \int_{0}^{1} u_{1}^{2} d x+\frac{R}{2} \int_{0}^{1} D^{2} u_{0}^{2}+\int_{0}^{1} F\left(D u_{0}\right) d x \\
& \leq \beta
\end{aligned}
$$

From (3.8) we can see that $S(t)$ maps $\left(u, u_{t}\right)$ from $H_{\beta}$ into itself and stay there forever. Moreover, we obtain

$$
\left\|u_{t}\right\| \leq C, \quad\left\|u_{x x}\right\| \leq C, \quad\left\|u_{x}\right\|_{L^{\infty}} \leq C
$$

and

$$
\int_{0}^{t}\left\|u_{t}\right\|^{2} d \tau \leq C, \quad \int_{0}^{t}\left\|u_{t}\right\|^{n+2} d \tau \leq C, \quad \forall n>0
$$

Second, we differentiate (1.4) with respect to $t$, multiply the resultant by $u_{t t}$, and integrate with respect to $x$ over $\Omega$ to obtain

$$
\frac{1}{2} \frac{d}{d t} \int_{0}^{1} u_{t t}^{2} d x+\mu \int_{0}^{1} u_{t t}^{2} d x+\int_{0}^{1} f\left(u_{x}\right)_{t} \cdot u_{x t t} d x+\frac{R}{2} \frac{d}{d t} \int_{0}^{1} u_{x x t}^{2} d x=0 .
$$

Here

$$
\int_{0}^{1} f\left(u_{x}\right)_{t} \cdot u_{x t t} d x=\int_{0}^{1} 5 \alpha_{1} u_{x}^{4} u_{x t} u_{x t t} d x-\int_{0}^{1} 3 \alpha_{2} u_{x}^{2} u_{x t} u_{x t t} d x-\int_{0}^{1} \alpha_{3} u_{x t} u_{x t t} d x
$$

In what follows, we estimate the right-hand side of (3.12). 
Since

$$
\int_{0}^{1} 5 \alpha_{1} u_{x}^{4} u_{x t} u_{x t t} d x=\frac{1}{2}\left(\frac{d}{d t} \int_{0}^{1} 5 \alpha_{1} u_{x}^{4} u_{x t}^{2} d x-\int_{0}^{1} 20 \alpha_{1} u_{x}^{3} u_{x t}^{3} d x\right)
$$

and from the estimates in (3.9), we have

$$
\left|\int_{0}^{1} u_{x}^{3} u_{x t}^{3} d x\right| \leq C \int_{0}^{1}\left|u_{x t}\right|^{3} d x
$$

Using Nirenberg's inequality yields

$$
\left\|u_{x t}\right\|_{L^{3}}^{3} \leq C\left\|u_{x x t}\right\|_{L^{2}}^{\frac{7}{4}} \cdot\left\|u_{t}\right\|_{L^{2}}^{\frac{5}{4}} \leq \delta\left\|u_{x x t}\right\|_{L^{2}}^{2}+C_{\delta}\left\|u_{t}\right\|_{L^{2}}^{10}
$$

with $\delta$ being a positive constant again.

In a similar manner we have

$$
\int_{0}^{1} 3 \alpha_{2} u_{x}^{2} u_{x t} u_{x t t} d x=\frac{1}{2}\left(\frac{d}{d t} \int_{0}^{1} 3 \alpha_{2} u_{x}^{2} u_{x t}^{2} d x-\int_{0}^{1} 6 \alpha_{2} u_{x} u_{x t}^{3} d x\right)
$$

and

$$
\left|\int_{0}^{1} u_{x} u_{x t}^{3} d x\right| \leq C \int_{0}^{1}\left|u_{x t}\right|^{3} d x \leq \delta\left\|u_{x x t}\right\|_{L^{2}}^{2}+C_{\delta}\left\|u_{t}\right\|_{L^{2}}^{10} .
$$

Therefore, we infer from (3.11) and the above estimates that

$$
\begin{aligned}
& \frac{d}{d t}\left(\frac{1}{2} \int_{0}^{1} u_{t t}^{2} d x+\frac{R}{2} \int_{0}^{1} u_{x x t}^{2} d x+\frac{5 \alpha_{1}}{2} \int_{0}^{1} u_{x}^{4} u_{x t}^{2} d x-\frac{3 \alpha_{2}}{2} \int_{0}^{1} u_{x}^{2} u_{x t}^{2} d x-\frac{\alpha_{3}}{2} \int_{0}^{1} u_{x t}^{2} d x\right) \\
& +\mu \int_{0}^{1} u_{t t}^{2} d x \leq \delta\left\|u_{x x t}\right\|_{L^{2}}^{2}+C_{\delta}\left\|u_{t}\right\|_{L^{2}}^{10} .
\end{aligned}
$$

Finally, we differentiate (1.4) with respect to $t$, multiply the resultant by $u_{t}$, and integrate with respect to $x$ over $\Omega$ to obtain

$$
\frac{d}{d t} \int_{0}^{1} u_{t} u_{t t} d x+\frac{\mu}{2} \frac{d}{d t} \int_{0}^{1} u_{t}^{2} d x+R \int_{0}^{1} u_{x x t}^{2} d x-\int_{0}^{1} u_{t t}^{2} d x+\int_{0}^{1} f\left(u_{x}\right)_{t} \cdot u_{x t} d x=0 .
$$

Here

$$
\begin{aligned}
\left|\int_{0}^{1} f\left(u_{x}\right)_{t} \cdot u_{x t} d x\right| & =\left|\int_{0}^{1} f^{\prime}\left(u_{x}\right) \cdot u_{x t}^{2} d x\right| \\
& \leq C\left\|u_{x t}\right\|_{L^{2}}^{2} \\
& \leq \delta\left\|u_{x x t}\right\|_{L^{2}}^{2}+C_{\delta}\left\|u_{t}\right\|_{L^{2}}^{2}
\end{aligned}
$$

Now we multiply (3.15) by $\frac{\mu}{2}$ and add the result to (3.14) to obtain

$$
\begin{aligned}
& \frac{d}{d t}\left(\frac{1}{2} \int_{0}^{1} u_{t t}^{2} d x+\frac{R}{2} \int_{0}^{1} u_{x x t}^{2} d x+\frac{5 \alpha_{1}}{2} \int_{0}^{1} u_{x}^{4} u_{x t}^{2} d x-\frac{3 \alpha_{2}}{2} \int_{0}^{1} u_{x}^{2} u_{x t}^{2} d x-\frac{\alpha_{3}}{2} \int_{0}^{1} u_{x t}^{2} d x\right. \\
& \left.+\frac{\mu}{2} \int_{0}^{1} u_{t} u_{t t} d x+\frac{\mu}{2} \int_{0}^{1} u_{t}^{2} d x\right)+\frac{\mu^{2}}{4} \int_{0}^{1} u_{t t}^{2} d x+\frac{\mu R}{2} \int_{0}^{1} u_{x x t}^{2} d x
\end{aligned}
$$




$$
\leq \delta\left\|u_{x x t}\right\|^{2}+C_{\delta}\left\|u_{t}\right\|^{2}
$$

Choosing $\delta$ small enough, we finally have

$$
\begin{aligned}
& \frac{d}{d t}\left(\frac{1}{2} \int_{0}^{1} u_{t t}^{2} d x+\frac{R}{2} \int_{0}^{1} u_{x x t}^{2} d x+\frac{5 \alpha_{1}}{2} \int_{0}^{1} u_{x}^{4} u_{x t}^{2} d x-\frac{3 \alpha_{2}}{2} \int_{0}^{1} u_{x}^{2} u_{x t}^{2} d x-\frac{\alpha_{3}}{2} \int_{0}^{1} u_{x t}^{2} d x\right. \\
& \left.+\frac{\mu}{2} \int_{0}^{1} u_{t} u_{t t} d x+\frac{\mu}{2} \int_{0}^{1} u_{t}^{2} d x\right)+\frac{\mu^{2}}{4} \int_{0}^{1} u_{t t}^{2} d x+\frac{\mu R}{4} \int_{0}^{1} u_{x x t}^{2} d x \leq C .
\end{aligned}
$$

If we define

$$
\begin{aligned}
E_{1}(t):= & \frac{1}{2} \int_{0}^{1} u_{t t}^{2} d x+\frac{R}{2} \int_{0}^{1} u_{x x t}^{2} d x+\frac{5 \alpha_{1}}{2} \int_{0}^{1} u_{x}^{4} u_{x t}^{2} d x-\frac{3 \alpha_{2}}{2} \int_{0}^{1} u_{x}^{2} u_{x t}^{2} d x-\frac{\alpha_{3}}{2} \int_{0}^{1} u_{x t}^{2} d x \\
& +\frac{\mu}{2} \int_{0}^{1} u_{t} u_{t t} d x+\frac{\mu^{2}}{4} \int_{0}^{1} u_{t}^{2} d x
\end{aligned}
$$

and

$$
E_{2}(t):=\frac{\mu}{2} \int_{0}^{1} u_{t t}^{2} d x+\frac{\mu R}{4} \int_{0}^{1} u_{x x t}^{2} d x .
$$

Combing the estimates obtained in (3.9), (3.10) with the equation (1.4), we get

$$
E_{1}(t) \sim\|u\|_{H^{4}}^{2}+\left\|u_{t}\right\|_{H^{2}}^{2}
$$

and

$$
E_{1}(t) \leq C E_{2}(t)
$$

Therefore

$$
\frac{d E_{1}(t)}{d t}+C_{1} E_{1}(t) \leq C_{2}
$$

then it immediately leads to

$$
E_{1}(t) \leq E_{1}(0) e^{-C_{1} t}+\frac{C_{2}}{C_{1}}
$$

It is clearly that here $C_{1}, C_{2}$ are positive constants depending only on $\beta$. Then we have for any initial data starting from any bounded set $\mathrm{B}$ of $H_{\beta}$, there exists some time $t_{0}(B)$, such that when $t \geq t_{0}(B)$,

$$
E_{1}(t) \leq \frac{2 C_{2}}{C_{1}}
$$

The existence of an absorbing set follows. The proof is complete.

Next, we focus on proving the uniform compactness of the orbits. For this we have to estimate higher-order derivatives. From now on we assume that the initial data belong to a bounded set B contained in $H_{\beta}$ and we use $\mathrm{C}, \tilde{C}$ to denote positive constants depending on $\mathrm{B}$ and $\beta$, i.e., $\left\|u_{0}\right\|_{H^{4}},\left\|u_{1}\right\|_{H^{2}}$ and $\beta$. 
Lemma 3.5. There exists some time $t_{1}=t_{1}(B)>0$, such that $\left(u, u_{t}\right)$ is bounded in $C\left(\left[t_{1},+\infty\right) ; H^{5} \times H^{3}\right)$.

Proof. First, we differentiate (1.4) with respect to $t$, multiply the resultant by $-u_{x x t t}$, and integrate with respect to $x$ over $\Omega$ to obtain

$$
\frac{d}{d t} \int_{0}^{1}\left(\frac{R}{2} u_{x x x t}^{2}+\frac{1}{2} u_{x t t}^{2}\right) d x+\frac{\mu}{2} \int_{0}^{1} u_{x t t}^{2} d x+\int_{0}^{1} f\left(u_{x}\right)_{x t} \cdot u_{x x t t} d x=0 .
$$

Multiplying (3.20) by t yields

$$
\begin{aligned}
& \frac{d}{d t}\left(\frac{1}{2} t\left\|u_{x t t}\right\|^{2}+\frac{R}{2} t\left\|u_{x x x t}\right\|^{2}\right)+\frac{\mu t}{2} \int_{0}^{1} u_{x t t}^{2} d x \\
& \leq \frac{1}{2}\left\|u_{x t t}\right\|^{2}+\frac{R}{2}\left\|u_{x x x t}\right\|^{2}+t \int_{0}^{1} f\left(u_{x}\right)_{x x t} \cdot u_{x t t} d x .
\end{aligned}
$$

Next, we differentiate (1.4) with respect to $t$, multiply the resultant by $-u_{x x t}$ and integrate with respect to $x$ over $\Omega$ to obtain

$$
\frac{\mu}{2} \frac{d}{d t} \int_{0}^{1} u_{x t}^{2} d x-\frac{d}{d t} \int_{0}^{1} u_{t t} \cdot u_{x x t} d x+R \int_{0}^{1} u_{x x x t}^{2} d x-\int_{0}^{1} u_{x t t}^{2} d x+\int_{0}^{1} f\left(u_{x}\right)_{x t} \cdot u_{x x t} d x=0
$$

Observe that if we integrate (3.17) with respect t, we arrive at

$$
\left\|u_{t t}\right\| \leq C, \quad\left\|u_{x x t}\right\| \leq C, \quad \int_{0}^{t}\left\|u_{t t}\right\|^{2} d \tau \leq C, \quad \int_{0}^{t}\left\|u_{x x t}\right\|^{2} d \tau \leq C
$$

with $C=C\left(\left\|u_{0}\right\|_{H^{4}},\left\|u_{1}\right\|_{H^{2}}\right)$. From (3.10), we also have

$$
\int_{0}^{t}\left\|u_{t}\right\|^{2} d \tau \leq C
$$

Using Nirenberg's inequality and equation (1.4), we have

$$
\int_{0}^{t}\left\|u_{x t}\right\|^{2} d \tau \leq C, \quad\left\|u_{x x x x}\right\| \leq C, \quad\left\|u_{x x x}\right\| \leq C .
$$

Then we integrate (3.22) with respect to t to arrive at

$$
\begin{aligned}
& R \int_{0}^{t}\left\|u_{x x x t}\right\|^{2} d \tau+\int_{0}^{t} \int_{0}^{1} f\left(u_{x}\right)_{x t} \cdot u_{x x t} d x d \tau+\frac{\mu}{2} \int_{0}^{1} u_{x t}^{2} d x-\left.\frac{\mu}{2} \int_{0}^{1} u_{x t}^{2}\right|_{t=0} d x \\
& =\int_{0}^{1} u_{t t} u_{x x t} d x-\left.\int_{0}^{1} u_{t t} u_{x x t}\right|_{t=0} d x+\int_{0}^{t}\left\|u_{x t t}\right\|^{2} d \tau
\end{aligned}
$$

Combing the estimates obtained in Lemma 3.4. and (3.23), we have

$$
\int_{0}^{t} \int_{0}^{1} f\left(u_{x}\right)_{x t} \cdot u_{x x t} d x d \tau
$$




$$
\begin{aligned}
& =\int_{0}^{t} \int_{0}^{1}\left(5 \alpha_{1} u_{x}^{4}-30 \alpha_{2} u_{x}^{2}-\alpha_{3}\right) u_{x x t}^{2} d x d \tau \\
& +\int_{0}^{t} \int_{0}^{1}\left(20 \alpha_{1} u_{x}^{3} u_{x t} u_{x x}-6 \alpha_{2} u_{x} u_{x t} u_{x x}\right) u_{x x t} d x d \tau \\
& \leq C \int_{0}^{t}\left\|u_{x x t}\right\|^{2} d \tau+C \leq C .
\end{aligned}
$$

Thus, it follows from (3.25)

$$
\int_{0}^{t}\left\|u_{x x x t}\right\|^{2} d \tau \leq C \int_{0}^{t}\left\|u_{x t t}\right\|^{2} d \tau+C
$$

Similarly, we also have

$$
\int_{0}^{t}\left\|u_{x t t}\right\|^{2} d \tau \leq C \int_{0}^{t}\left\|u_{x x x t}\right\|^{2} d \tau+C .
$$

In the follows, we estimate the last term of the right-hand side of (3.21).

Since

$$
f\left(u_{x}\right)_{x t}=20 \alpha_{1} u_{x}^{3} u_{x t} u_{x x}+5 \alpha_{1} u_{x}^{4} u_{x x t}-3 \alpha_{2} u_{x}^{2} u_{x x t}-6 \alpha_{2} u_{x} u_{x t} u_{x x}-\alpha_{3} u_{x x t}
$$

Here

$$
\left|\int_{0}^{t} \int_{0}^{1}\left(20 \alpha_{1} u_{x}^{3} u_{x t} u_{x x}\right)_{x} \cdot u_{x t t} d x d \tau\right| \leq \delta \int_{0}^{t}\left\|u_{x t t}\right\|^{2} d \tau+C_{\delta} \int_{0}^{t} \int_{0}^{1}\left(u_{x}^{3} u_{x t} u_{x x}\right)_{x}^{2} d x d \tau
$$

and

$$
\begin{aligned}
\int_{0}^{t} \int_{0}^{1}\left(u_{x}^{3} u_{x t} u_{x x}\right)_{x}^{2} d x d \tau & =\int_{0}^{t} \int_{0}^{1}\left(3 u_{x}^{2} u_{x x}^{2} u_{x t}+u_{x}^{3} u_{x t} u_{x x x}+u_{x}^{3} u_{x x} u_{x x t}\right)^{2} d x d \tau \\
& \leq C \int_{0}^{t} \int_{0}^{1}\left(u_{x t}^{2}+u_{x x t}^{2}\right) d x d \tau \leq C
\end{aligned}
$$

Thus, we have

$$
\left|\int_{0}^{t} \int_{0}^{1}\left(20 \alpha_{1} u_{x}^{3} u_{x t} u_{x x}\right)_{x} \cdot u_{x t t} d x d \tau\right| \leq \delta \int_{0}^{t}\left\|u_{x t t}\right\|^{2} d \tau+C_{\delta}
$$

In a similar manner to (3.30), we have

$$
\left|\int_{0}^{t} \int_{0}^{1}\left(6 \alpha_{2} u_{x} u_{x t} u_{x x}\right)_{x} \cdot u_{x t t} d x d \tau\right| \leq \delta \int_{0}^{t}\left\|u_{x t t}\right\|^{2} d \tau+C_{\delta}
$$

And

$$
\begin{aligned}
\int_{0}^{1}\left(5 \alpha_{1} u_{x}^{4} u_{x x t}\right)_{x} \cdot u_{x t t} d x & =-\int_{0}^{1}\left(5 \alpha_{1} u_{x}^{4} u_{x x t}\right) \cdot u_{x x t t} d x \\
& =-\frac{1}{2}\left(\frac{d}{d t} \int_{0}^{1} 5 \alpha_{1} u_{x}^{4} u_{x x t}^{2} d x-\int_{0}^{1} 20 \alpha_{1} u_{x}^{3} u_{x x t}^{3} d x\right)
\end{aligned}
$$


By Nirenberg's inequality and Young's inequality, we find that

$$
\left|\int_{0}^{t} \int_{0}^{1} 20 \alpha_{1} u_{x}^{3} u_{x x t}^{3} d x d \tau\right| \leq C \int_{0}^{t} \int_{0}^{1}\left|u_{x x t}\right|^{3} d x d \tau
$$

and

$$
\begin{gathered}
\left\|u_{x x t}\right\|_{L^{3}} \leq C\left\|u_{x x x t}\right\|_{L^{2}}^{\frac{1}{6}} \cdot\left\|u_{x x t}\right\|_{L^{2}}^{\frac{5}{6}}, \\
\left\|u_{x x t}\right\|_{L^{3}}^{3} \leq C\left\|u_{x x x t}\right\|_{L^{2}}^{\frac{1}{2}} \cdot\left\|u_{x x t}\right\|_{L^{2}}^{\frac{5}{2}} \\
\leq \delta\left\|u_{x x x t}\right\|_{L^{2}}^{2}+C_{\delta}\left\|u_{x x t}\right\|_{L^{2}}^{\frac{10}{3}} .
\end{gathered}
$$

Thus,

$$
\int_{0}^{t} \int_{0}^{1}\left|u_{x x t}\right|^{3} d x d \tau \leq \delta \int_{0}^{t}\left\|u_{x x x t}\right\|^{2} d \tau+C_{\delta}
$$

Similarly, we have

$$
\begin{aligned}
-\int_{0}^{1}\left(3 \alpha_{2} u_{x}^{2} u_{x x t}\right)_{x} \cdot u_{x t t} d x & =\int_{0}^{1}\left(3 \alpha_{2} u_{x}^{2} u_{x x t}\right) \cdot u_{x x t t} d x \\
& =\frac{1}{2}\left(\frac{d}{d t} \int_{0}^{1} 3 \alpha_{2} u_{x}^{2} u_{x x t}^{2} d x-\int_{0}^{1} 6 \alpha_{2} u_{x} u_{x x t}^{3} d x\right),
\end{aligned}
$$

and

$$
\left|\int_{0}^{t} \int_{0}^{1} 6 \alpha_{2} u_{x} u_{x x t}^{3} d x d \tau\right| \leq \delta \int_{0}^{t}\left\|u_{x x x t}\right\|^{2} d \tau+C_{\delta} .
$$

Finally, we deduce

$$
\int_{0}^{t} \int_{0}^{1} f\left(u_{x}\right)_{x x t} \cdot u_{x t t} d x d \tau \leq \delta \int_{0}^{t}\left\|u_{x t t}\right\|^{2} d \tau+\delta \int_{0}^{t}\left\|u_{x x x t}\right\|^{2} d \tau+\tilde{C}_{\delta} .
$$

with $\tilde{C}_{\delta}=\tilde{C}\left(\left\|u_{0}\right\|_{H^{4}},\left\|u_{1}\right\|_{H^{2}}, \delta\right)$.

Now we integrate (3.21) with respect t to obtain

$$
\begin{aligned}
& \frac{1}{2} t\left\|u_{x t t}\right\|^{2}+\frac{R}{2} t\left\|u_{x x x t}\right\|^{2}+\frac{\mu}{2} \int_{0}^{t} \tau\left\|u_{x t t}\right\|^{2} d \tau \\
& \leq \frac{1}{2} \int_{0}^{t}\left\|u_{x t t}\right\|^{2} d \tau+\frac{R}{2} \int_{0}^{t}\left\|u_{x x x t}\right\|^{2} d \tau+\delta t \int_{0}^{t}\left\|u_{x t t}\right\|^{2}+\left\|u_{x x x t}\right\|^{2} d \tau+\tilde{C}_{\delta} t .
\end{aligned}
$$

Combing (3.40) with (3.27), (3.28) for any $t \geq 1$ and choosing $\delta \ll \mu$ small enough yields

$$
\frac{1}{2}\left\|u_{x t t}\right\|^{2}+\frac{R}{2}\left\|u_{x x x t}\right\|^{2} \leq \frac{1}{2} \int_{0}^{t}\left\|u_{x t t}\right\|^{2} d \tau+\frac{R}{2} \int_{0}^{t}\left\|u_{x x x t}\right\|^{2} d \tau+C t .
$$

Using Gronwall's inequality yields

$$
\frac{1}{2}\left\|u_{x t t}\right\|^{2}+\frac{R}{2}\left\|u_{x x x t}\right\|^{2} \leq C t \cdot e^{t} .
$$


Let $t=1$ in (3.42) to obtain

$$
\frac{1}{2}\left\|\left.u_{x t t}\right|_{t=1}\right\|^{2}+\frac{R}{2}\left\|\left.u_{x x x t}\right|_{t=1}\right\|^{2} \leq C e
$$

with $C=C\left(\left\|u_{0}\right\|_{H^{4}},\left\|u_{1}\right\|_{H^{2}}\right)$.

Integrating (3.21) again with respect $\mathrm{t}$ in $[1,+\infty)$, combing the the resultant with (3.27), (3.28) and (3.39), we derive that there exists $t_{1}>1$ large enough in (3.21), such that, when $t>t_{1}$ the terms on the right-hand side of (3.21), i.e.,

$\frac{1}{2} \int_{1}^{t}\left\|u_{x t t}\right\|^{2} d \tau+\frac{R}{2} \int_{1}^{t}\left\|u_{x x x t}\right\|^{2} d \tau+\delta t \int_{1}^{t}\left\|u_{x x x t}\right\|^{2}+\left\|u_{x t t}\right\|^{2} d \tau$ can be absorbed by $\frac{1}{4} \mu \int_{1}^{t} \tau\left\|u_{x t t}\right\|^{2} d \tau+C$. Then, we get

$$
\frac{1}{2} t\left\|u_{x t t}\right\|^{2}+\frac{R}{2} t\left\|u_{x x x t}\right\|^{2}+\frac{\mu}{4} \int_{1}^{t} \tau\left\|u_{x t t}\right\|^{2} d \tau \leq C+C e+C t, \text { for any } t \geq t_{1}
$$

with $C=C\left(\left\|u_{0}\right\|_{H^{4}},\left\|u_{1}\right\|_{H^{2}}\right)$.

Integrating (3.44) with respect to t yields

$$
\frac{1}{2}\left\|u_{x t t}\right\|^{2}+\frac{R}{2}\left\|u_{x x x t}\right\|^{2} \leq \frac{C}{t}+C, \text { for any } t \geq t_{1}
$$

Combing (3.45) with equation (1.4), we conclude our argument. The proof is complete.

From the last lemma the compactness of the orbit in $H^{4} \times H^{2}$ follows.

In a similar manner to Section 2, applying the Theorem by Temann again, which can be rephrased as follows, we deduce the results of Theorem 1.2. (ii).

Theorem 3.1. Suppose that

(a) the mapping $S(t), t \geq 0$ defined by the solution to problems (1.4)-(1.3) is a nonlinear continuous semigroup from $H$ into itself;

(b) the operators $S(t)$ are uniformly compact for $t$ large, i.e., for every bounded set $B$ contained in $H_{\beta}$, there exists $t_{1}$ which may depend on $B$ such that $\cup_{t \geq t_{1}} S(t) B$ is relatively compact in $H$;

(c) the orbit starting from any bounded set of $H_{\beta}$ will reenter in $H_{\beta}$ after a finite time, which depends only on this bounded set, and stay there forever; there exists a bounded set $B_{\beta}$ in $H_{\beta}$ such that $B_{\beta}$ is absorbing in $H_{\beta}$.

Then the $\omega$-limit set of $B_{\beta}, A_{\beta}$ is a global attractor which is compact and attracts the bounded sets of $H_{\beta}$.

Therefore, the proof of Theorem 1.2. (ii) is complete. 
Acknowledgement: The author Shang was partially supported by the program by China Scholarship Council and supported by the NSF of China under the grant No. 10371022, and also by Chinese Ministry of Education under the grant No. 20050246002. Shang also would like to take this opportunity to express her sincerely thanks to her supervisor Prof. Songmu Zheng, for his constant encouragement and supports.

\section{References}

[1] J.M.Ball, Initial-boundary value problems for an extensible beam, J.Math.Anal.Appl 42(1973), 61-90.

[2] J.M.Ball, Stability theory for an extensible beam, J.Differential Equations 14(1973), 399-418.

[3] F.Falk, Ginzberg-Landau theory of static domain walls in shape memory alloys, Physica B, 51(1983), 177-185.

[4] F.Falk, Ginzberg-Landau theory of solitary waves in shape memory alloys, Physica B, 54(1984), 159-167.

[5] A.Eden, A.J.Milani, Exponential attractors for extensible beam equation, Nonlinearity 6(1993), 457-479.

[6] W.Shen, S.Zheng, On the coupled Cahn-Hilliard equations, Comm.PDE 18(1993), 701-727.

[7] Z.Chen, K.H.Hoffmann, On a one-dimensional thermoviscoelastic model for structural phase transitions in shape memory alloys, J.Differential Equations 112(1994), 325-350.

[8] R.Racke, S.Zheng, Global existence and asymptotic behavior in nonlinear thermoviscoelasticity, J.Differential Equations 134(1997), 46-67.

[9] J.Sprekels, S.Zheng, Maximal attractor for the system of a Landau-Ginzburg theory for structural phase transitions in shape memory alloys, Physica D 121(1998), 252262.

[10] J.Sprekels, S.Zheng, P.Zhu, Asymptotic behavior of the solutions to a LandauGinzburg system with viscosity for martensitic phase transitions in shape memeory alloys, SIAM J.Math.Anal 29(1998), 69-84. 
[11] Y.Qin, H.Liu, C.Song, Global attractor for a nonlinear thermoviscoelastic system in shape memory alloys, Proc.Roy.Soc.Edinburgh A (To appear).

[12] C.Shang, Global Attractor for the Ginzburg-Landau Thermoviscoelastic Systems with Hinged Boundary Conditions, J.Math.Anal.Appl 343(2008), 1-21.

[13] J.K.Hale, Asymptotic Behavior of Dissipative Systems, AMS Mathematical Surveys and Monographs no.25 (Providence, RI: American Mathematical Society), 1988.

[14] R.Temam, Infinite-Dimensional Dynamical System in Mechanics and Physics, Applied Mathematics and Science, Vol. 68, Springer, New York, 1998.

[15] S.Jiang, R.Racke, Evolution Equations in Thermoelasticity, $\pi$ Monographs Surveys Pure Appl.Math. 112, Chapman Hall/CRC, Boca Raton, 2000.

[16] S.Zheng, Nonlinear Evolution Equations, Pitman Monographs and Surverys in Pure and Applied Mathematics, 133 Chapman Hall/CRC, BocaRaton, Florida, 2004.

Reinhard Racke, Department of Mathematics and Statistics, University of Konstanz,

78457 Konstanz, Germany

reinhard.racke@uni-konstanz.de

Chanyu Shang, Department of Mathematics and Statistics, University of Konstanz,

78457 Konstanz, Germany

on leave from

Institute of Mathematics, Fudan University, 200433 Shanghai, P.R.China

chanyufd@gmail.com 